\title{
LOS MÉTODOS DE ILUMINACIÓN DURANTE EL PALEOLITICO EN LA PENÍNSULA IBÉRICA ${ }^{29}$
}

\author{
The Illumination Methods during the Paleolithic period in the \\ Iberian Peninsula
}

Lucía Esperanza Doral Chapado¡Error! Marcador no definido. ${ }^{30}$

\section{Resumen}

El descubrimiento del fuego por los hombres prehistóricos supuso un cambio sustancial en la vida de estos hombres, de sus costumbres y usos diarios como la defensa contra los animales salvajes, los cambios producidos en su alimentación, e incluso una forma de combatir el frio. Además, fue un elemento indispensable para poder combatir la oscuridad dentro de las cuevas y grutas donde se refugiaron. A pesar de ello, no son muchos los historiadores que han profundizado en el estudio de la iluminación y su relación con el arte rupestre; cabe mencionar entre otros a María Ángeles Medina; Araceli Cristo; Antonio Romero, José Luis Sanchidrián o Sophie A. de Beaune.

La aparición de las lámparas hace 40.000 años posiblemente favoreció la aparición del "arte" en cuevas y grutas, pinturas, adornos personales, armas más complejas, etc. Por eso es imprescindible conocer qué tipo de combustible y de mecha era el más idóneo para la fabricación de estas lámparas.

En el presente trabajo se ha estudiado la eficacia (duración e iluminación) de tres tipos de combustibles disponibles en aquella época: cera de abeja, sebo y tuétano, con la misma mecha y el mismo soporte-

Nuestros resultados indican que el combustible más idóneo en las condiciones del experimento sería el tuétano.

Palabras clave: Paleolítico Superior, lámparas, combustible, mecha.

\begin{abstract}
The discovery of fire brought with it a considerable change in the lives of those men across several aspects in their customs and daily uses such as defense against wild animal, changes in their diet and even way to fight the cold. Nevertheless, without doubt, it was an essential element to combat the darkness inside the caves and grottos where they took refuge. However, there are not many historians who have given importance to the study of lighting and its relationship with cave painting. But, it is worth mentioning among others María Ángeles Medina, Araceli Cristo, Antonio Romero, José Luis Sanchidrián o Sophie A de Beaune.
\end{abstract}

The appearance of lamps 40.000 years ago was parallel to the appearance of art in caves and grottos, paintings, personal ornaments, weapons that are more complex, etc. That why it is essential to know what type of fuel and wick was the most suitable for manufacturing these lamps.

\footnotetext{
${ }^{29}$ Recibido: 22/12/2018. Aceptado: 10/01/2019. http://doi.org/10.15366/baexuam2018-19.13.007

30 Universidad Autónoma de Madrid. ORCID: 0000-0002-7047-2009. E-mail: lucia.doral@estudiante.uam.es
} 
In this work, we have studied the efficiency (length and illumination) of three kinds of fuel: bee wax, tallow and marrow, with the same wick and bracket.

Our results show that the ideal fuel in the conditions of the experiment is marrow.

Keywords: Upper Paleolithic, lamps, fuel, wick

\section{INTRODUCCIÓN}

Con este trabajo pretendemos dar un poco de luz a un hecho fundamental que ha pasado inadvertido para muchos historiadores, como fue el descubrimiento del fuego por los hombres prehistóricos. Este gran descubrimiento permitió a estos hombres un cambio sustancial en sus vidas, abarcando varias facetas en sus costumbres y usos diarios como la defensa contra los animales salvajes, los cambios producidos en su alimentación, e incluso una forma de combatir el frio. Pero sin duda fue un elemento indispensable para poder combatir la oscuridad dentro de las cuevas y grutas donde se refugiaron. A pesar de ello, no son muchos los historiadores que han profundizado en el estudio de la iluminación y su relación con el arte rupestre; cabe mencionar entre otros a María Ángeles Medina; Araceli Cristo; Antonio Romero, José Luis Sanchidrián o Sophie A. de Beaune.

La aparición de las lámparas hace aproximadamente 40.000 años fue paralela a uno de los cambios culturales más importantes que se produjeron en la historia, la aparición del "arte", pinturas, adornos personales, armas más complejas, etc. Es plausible pensar que la utilización de las lámparas favoreció el desarrollo del arte murario en las cuevas y grutas, ya que éstas aportaban luz en las profundidades de estos lugares. Por eso es imprescindible conocer qué tipo de combustible y de mecha era el más idóneo para la fabricación de estas lámparas.

Hay indicios del uso del tuétano como combustible, ya en el Paleolítico Superior para realizar las pinturas, pero se han hallado restos en Atapuerca que pueden datar del Paleolítico Medio o Inferior (Diario de los yacimientos de Atapuerca). Además, este combustible se transforma en una especie de aceite que no emite humo, convirtiéndose en un elemento imprescindible para asegurar el resultado de estos artistas rupestres. Dichas lámparas entraron a formar parte de los útiles comunes en la vida de estos hombres y mujeres prehistóricos.

Los estudios realizados en la cueva malagueña de Ardales por Pedro Cantalejo y María del Mar Espejo desvelan el uso frecuente de cera de abeja como combustible, que aporta una luz más duradera y clara, que la de grasa de animal (Caso, 2011).

Siguiendo los estudios de Sophie A. Beaune (1987:109) podemos destacar otros tipos de combustible utilizados para hacer lámparas en la prehistoria, entre ellos; minerales (carbón, lignitos y petróleo); vegetales (árboles, arbustos, algas, turbas, raíces, resina y aceites de frutas).

Aunque es un misterio aún sin resolver lo que llevó a los hombres de la prehistoria a fabricar antorchas y lámparas con un hueso de un animal y una mecha, hoy sabemos por qué funcionó: la grasa del tuétano se funde con el calor y sube por la mecha por capilaridad. Parte de esa grasa que asciende se transforma en gas, y este arde en el extremo de la mecha, haciendo que la llama perdure durante un tiempo. Para determinar la mecha que se utilizaba, se han analizado los residuos de las lámparas y se han encontrado restos de coníferas, enebro, hierbas o liquen y musgo. 
Estos útiles nos han llegado en forma de materiales arqueológicos, que nos acercan a un conocimiento mayor, y nos ayudan a comprender mejor algunos de los aspectos más complejos de la espiritualidad e imaginario de este periodo. Este invento supuso un acicate en la búsqueda sobre todo de lugares adecuados para la realización de sus pinturas parietales. No podemos olvidar que la mayoría de los descubrimientos de los paneles más impresionantes se ubican en la profundidad de las cavidades, en galerías y espacios sumidos en la total oscuridad y donde sería necesaria por tanto la luz artificial.

Los estudios realizados a este respecto distinguen entre tres estilos de iluminación en cuevas: lámparas de piedra, antorchas y foyers. Las lámparas de grasa animal pueden a su vez ser de circuito abierto o cerrado (Fig. 1). Las primeras están constituidas por superficies planas o leves concavidades, que no recogen todo el combustible en el soporte, sino que lo dejan fluir fuera del recipiente. Por el contrario, las segundas pueden o no tener un mango, pero su característica principal es una especie de cazuela, bien natural o trabajada (de Beaune, 2000)
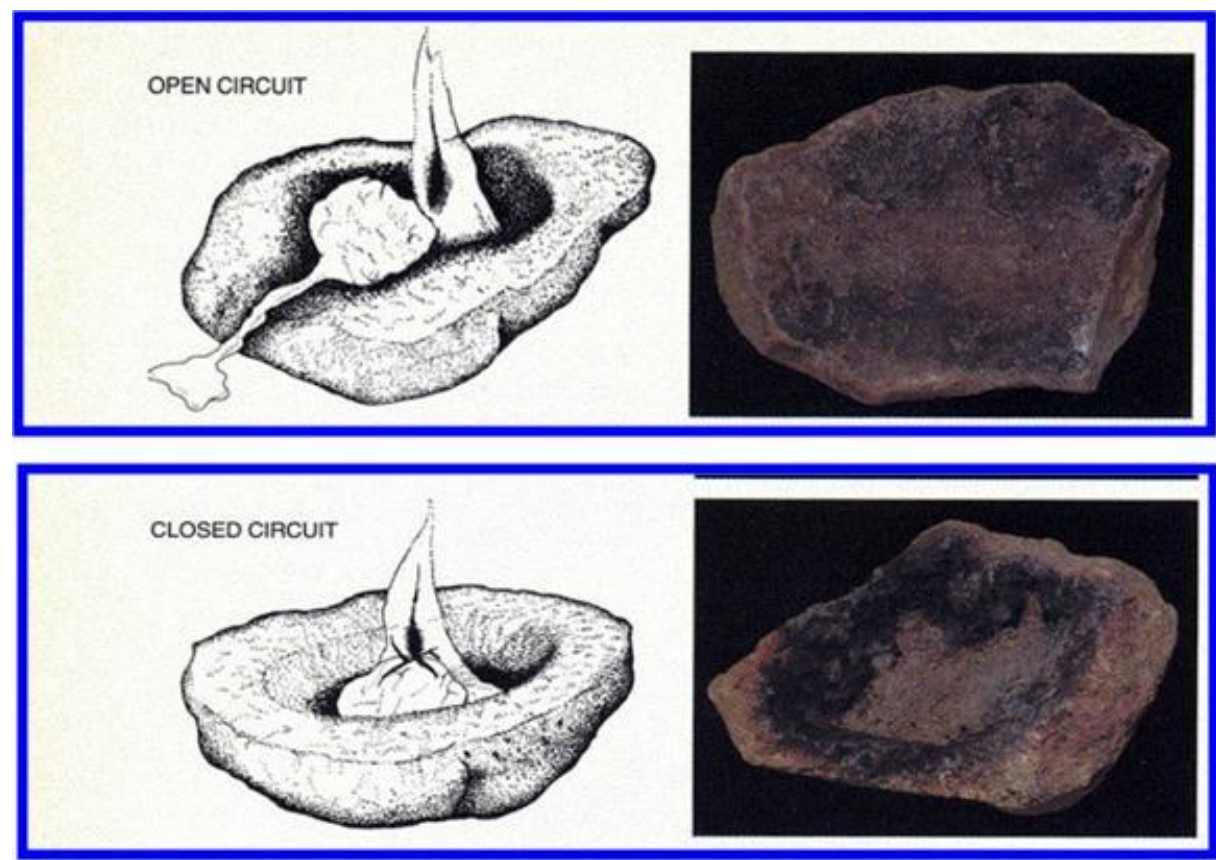

Figura 1. Lámparas de grasa animal.

Las que se han encontrado en la cueva de Llonin (Asturias) son de circuito cerrado sobre arenisca roja y son puntos de luz móviles (Fig. 2).

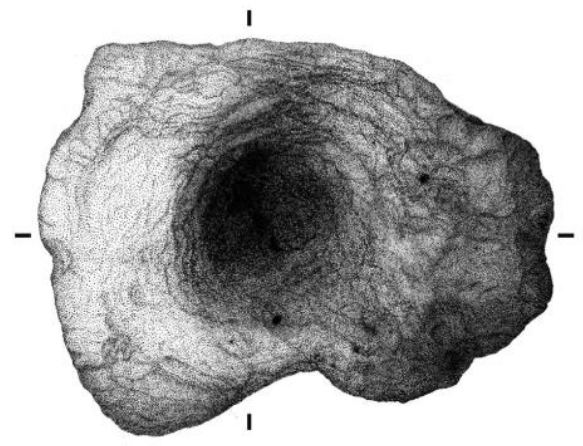

Figura 2. Lámpara de Llonin. Imagen obtenida de De la Rasilla et al., 2010. 
Con relacion a las antorchas, presentan la ventaja de dar más luz e iluminar en todas las direcciones y ademas iluminaban el suelo evitando que sufriesen accidentes al estar en lugares irregulares. Pero presentaban el incoveniente de durar menos tiempo y limitaba la movilidad del porteador.

"Los foyers son combustiones para iluminar y alimentar las lámparas y antorchas utilizadas durante el tránsito por la cavidad; se diferencian de los hogares en los restos que contiene en el interior, en su exclusiva utilización de alumbrado y, por lo general, se encuentran en lugares más profundos de la cavidad y sus restos se hallan más dispersos" (Medina et al., 2012:107).

El mayor número de lámparas se han encontrado en cuevas del norte peninsular: Llonin; El Pendo; Tito Bustillo; Covarón, etc. (de la Rasilla et al., 2010) coincidiendo con las zonas donde hay una mayor concentracion de cuevas con pinturas rupestres. En cuanto al sur de la península, se han hallado lámparas en cuevas malagueñas, como por ejemplo, en Cueva de Ardales (Cantalejo et al., 2014), la Cueva de la Pileta (Cortés et al., 2016) y en cuevas granadinas como la Cueva del Malmamuerzo.

El objetivo del experimento que se propone hacer dentro del campo de la Arqueología Experimental es fabricar, de forma artesanal e imitando la técnica del Paleolítico, una lámpara de piedra con una convexidad para albergar el combustible y determinar qué tipo es el que proporciona una mayor intensidad de luz y cuál permite un tiempo mayor de uso, a tenor de los estudios previos sobre este tema expuestos en el inicio del trabajo. Hay que tener en cuenta que el tamaño de la lámpara fabricada es de una capacidad menor, en comparación con las que nos encontramos en los yacimientos.

- Las variables fijas son el soporte, la cantidad de combustible y el tamaño de la mecha.

- Las variables independientes son: la grasa de tuétano de un hueso de animal, el sebo de un animal y la cera virgen de abeja.

- Las variables dependientes son la intensidad de la luz y su tiempo de duración.

- La observación se hará a través de un luxómetro HANNA modelo HI 97500 del Laboratorio de Prehistoria y Arqueología de la Universidad Autónoma de Madrid (Fig. 3), al igual que la báscula de precisión que se utilizó para pesar los distintos combustibles. 


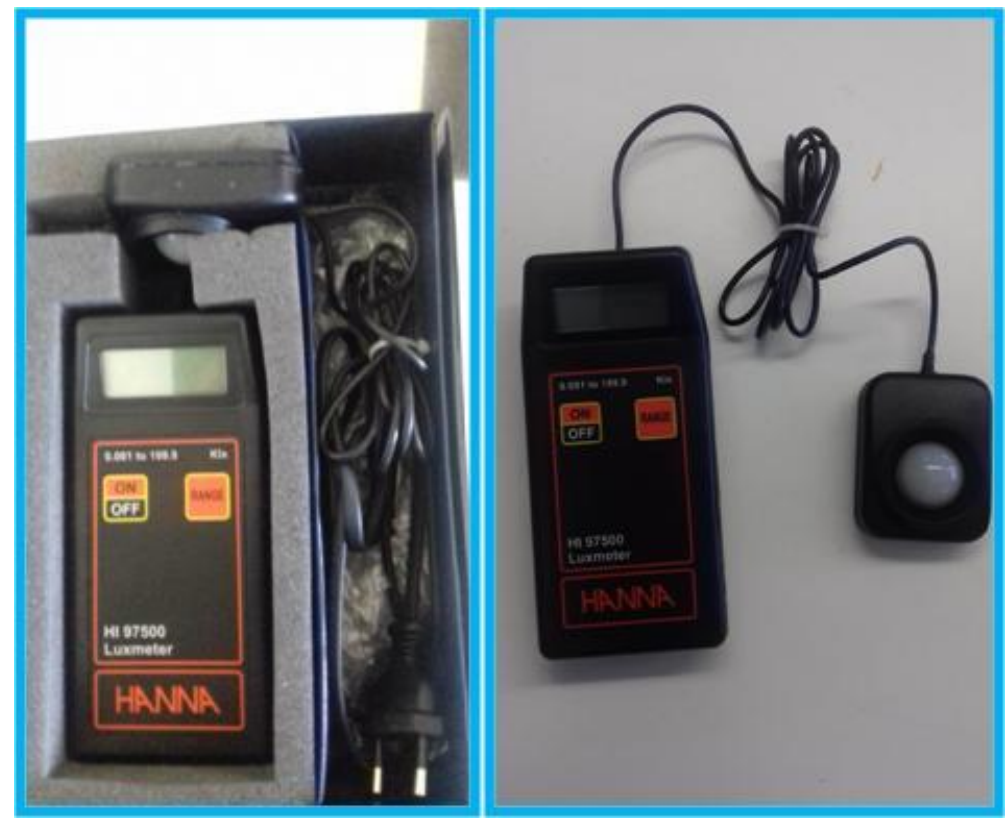

Figura 3. Luxómetros utilizados en el trabajo

\section{Experimento 1}

Cera de abeja y mecha de estopa.

\section{Experimento 2}

Tuétano y mecha de estopa

\section{Experimento 3}

Sebo de animal y mecha de estopa

\section{PROCEDIMIENTO}

Lo primero es proceder a la fabricación de la lámpara. Para ello se utiliza una piedra de arenisca del noroeste peninsular. Tras marcar con un lápiz el diámetro que necesitamos para depositar dentro el combustible, se pasa a hacer una concavidad mediante la técnica de repiqueteado y abrasión. Se utiliza un canto de cuarcita de río para esta última técnica y un percutor o pico de cuarcita procedente de afloramientos de Ciudad Real y otro de sílex del Mioceno de Madrid para la primera (Figs. 4 y 5). El tiempo de ejecución ha sido de 13 horas, en las cuales se han tenido que ir retocando las herramientas para sacar filo y facilitar el trabajo. 


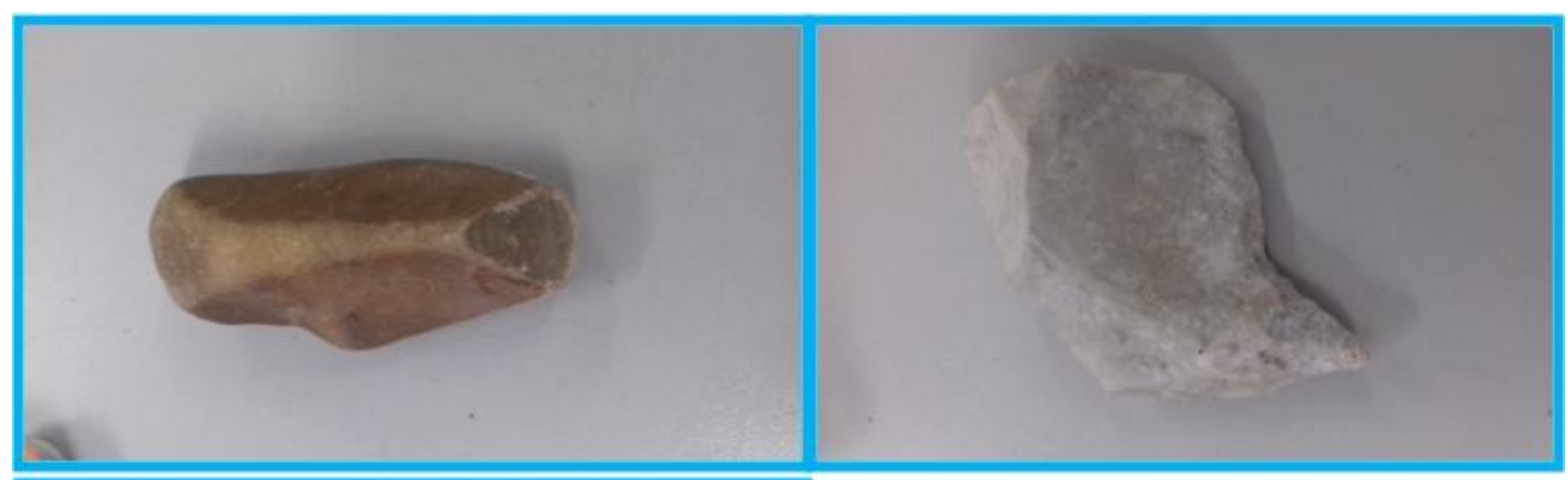

Figura 4. Herramientas empleadas
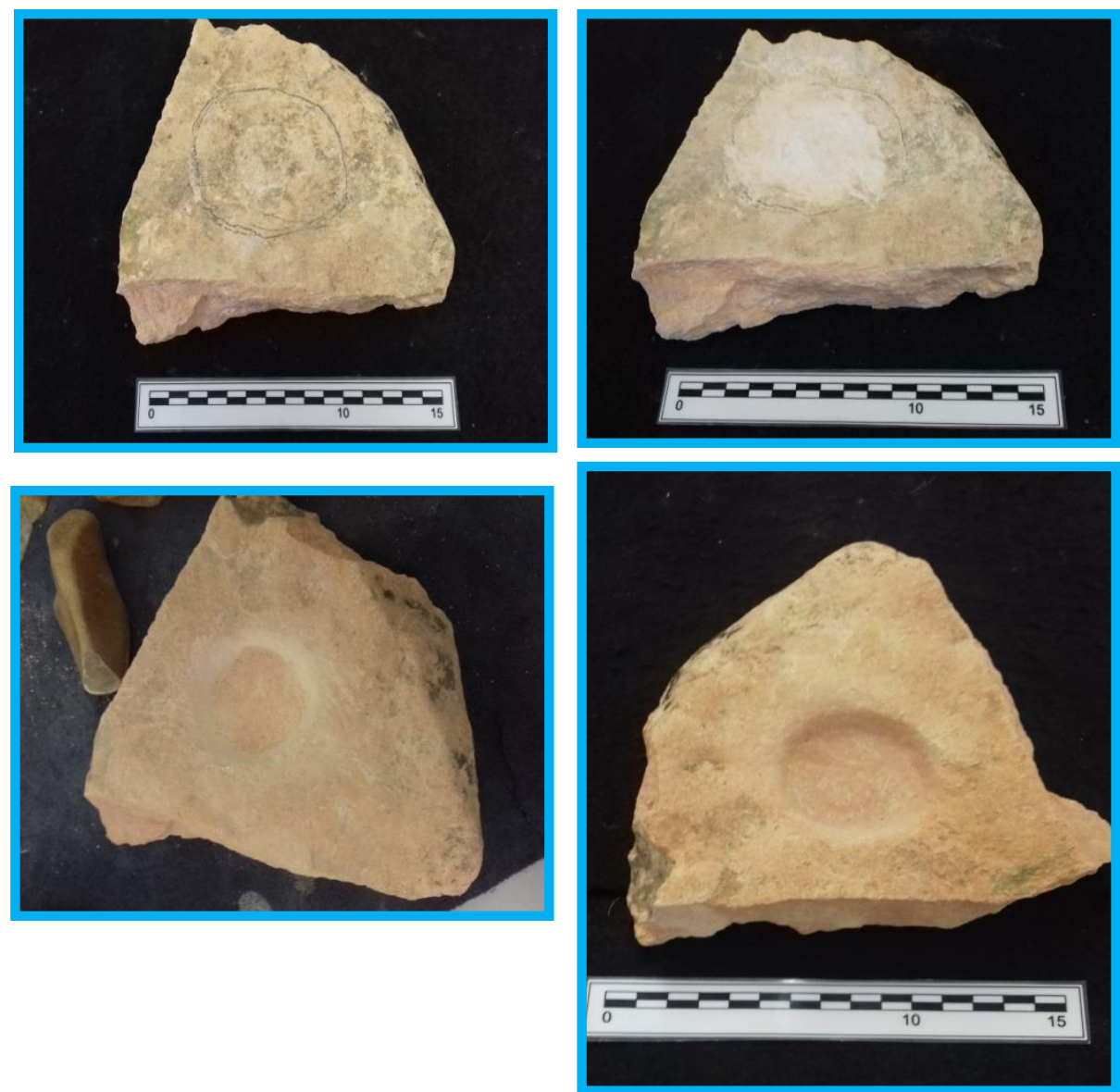

Figura 5. Secuencia de fabricación de la lámpara 
En segundo lugar, se han fabricado las tres mechas (Fig. 6). El material utilizado ha sido estopa, se han trenzado unos $6 \mathrm{~cm}$ de ella para cada mecha y se han engrasado con el tuétano.

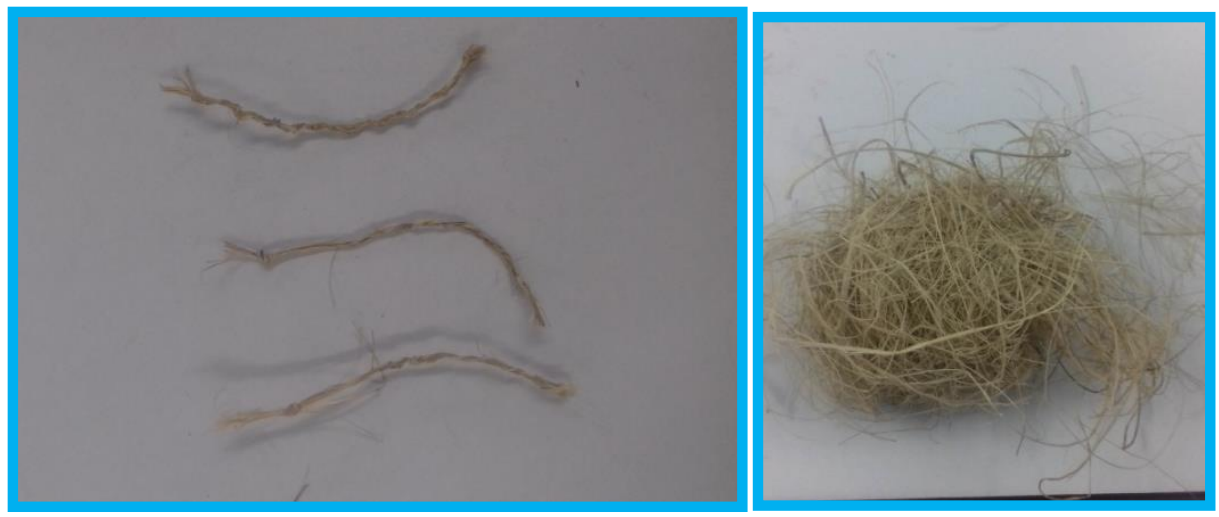

Figura 6. Mechas

En tercer lugar, procedimos a pesar $24 \mathrm{~g}$ de cada combustible (Fig. 7).

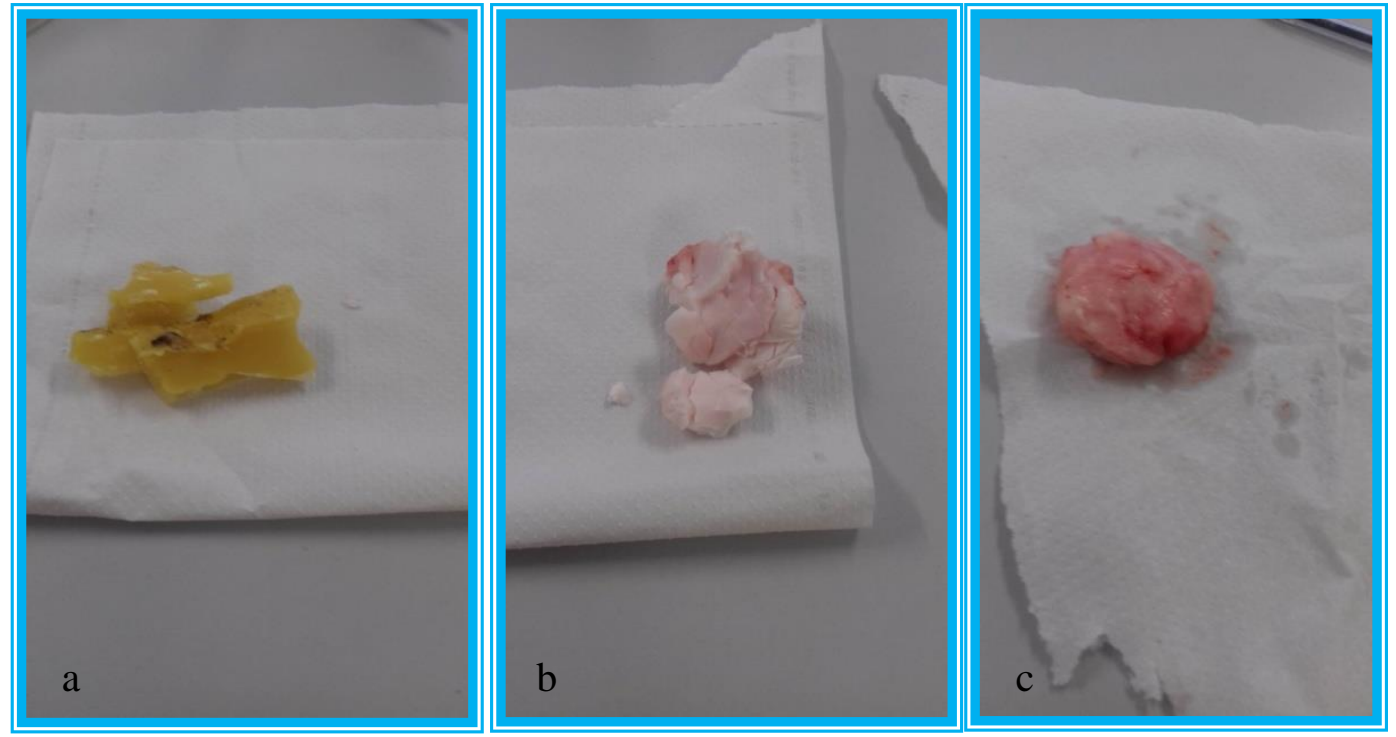

Figura 7: Combustibles: a) cera; b) tuétano; c) sebo

Por último, pasamos a ejecutar la preparación de cada combustible con su mecha. Para la lámpara de cera de abeja primero amasamos con las manos para calentar los $24 \mathrm{~g}$ de cera y ablandarla durante una hora, y después introducimos la mecha dejándola sobresalir unos dos centímetros. Para extraer el tuétano del hueso se ha utilizado un elemento afilado en la punta y, tras darle forma, se ha introducido la mecha dejando dos centímetros en la superficie. Para la lámpara de sebo ha sido necesario amasar también unos quince minutos antes de introducir la mecha.

\subsection{Primer experimento}

Con la lámpara de cera, en el momento de encender la mecha acercamos el luxómetro al borde de la lámpara y nos da 0,13 lx; volvemos a medir la intensidad lumínica a la media hora y nos da 0,03 lx; pasada una hora desde que se encendió la mecha, la llama es muy pequeña y apenas da luz, y se apaga pasadas dos horas y media desde su inicio, sin consumirse todo el combustible (Fig. 8). 


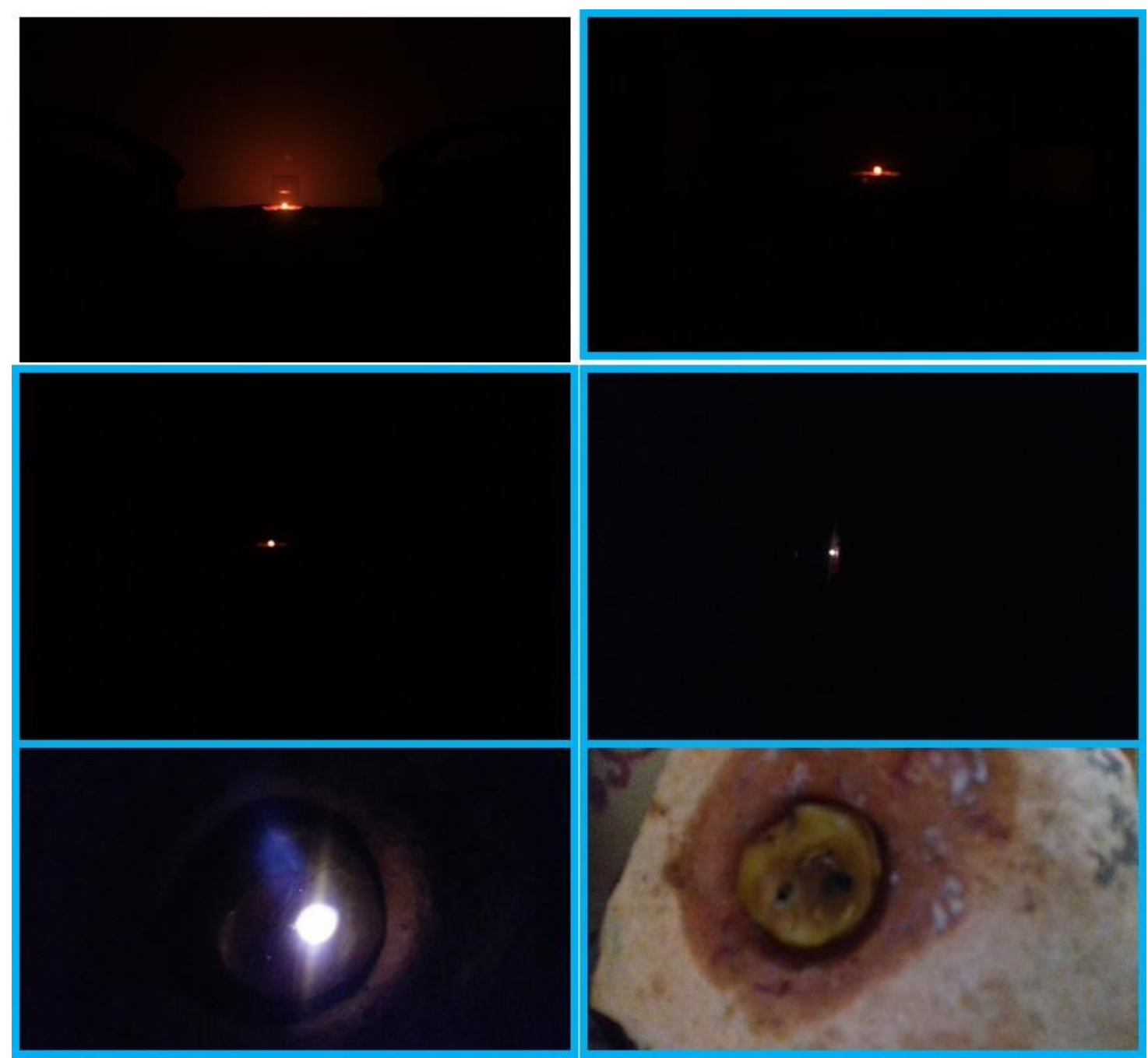

Figura 8. Lámpara de cera. Evolución de la iluminación

\subsection{Segundo experimento}

La lámpara de tuétano al inicio del experimento da 0,12 lx de intensidad lumínica. A la hora del inicio 0,02 lx. A las dos horas da 0,02 lx. A las tres horas 0,03/0,04 lx. A las cuatro horas da 0,10 lx. A las cuatro horas y media da 0,15 lx. A las cinco horas da 0,20/0,23 lx. A las cinco horas y media da 0,13 lx. A las seis horas da 0,10 lx. A las seis horas y diez minutos da 0,02 lx. Y a las seis horas y media se apaga (Figs. 9 y 10). 


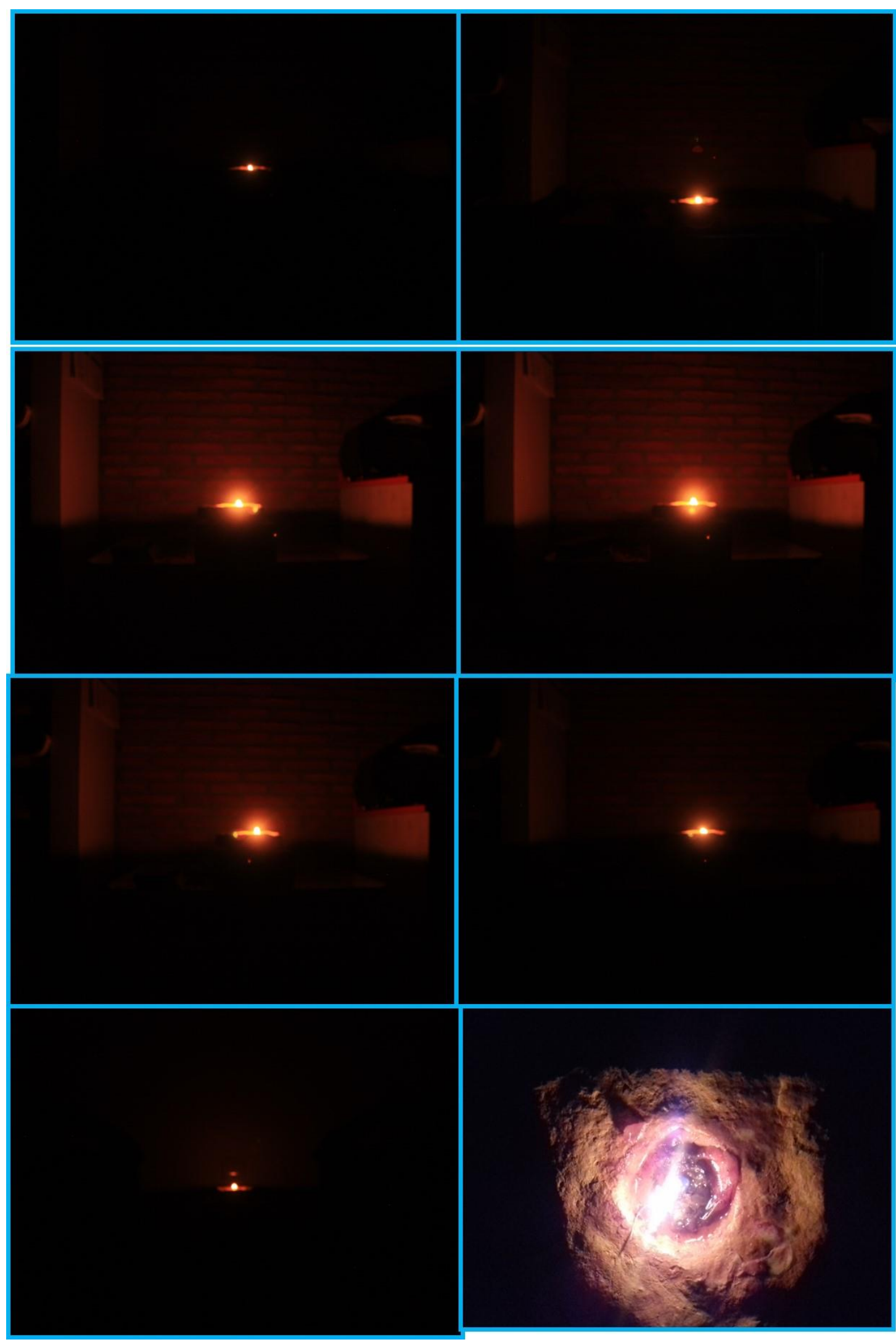

Figura 9: Lámpara de tuétano. Evolución de la iluminación 


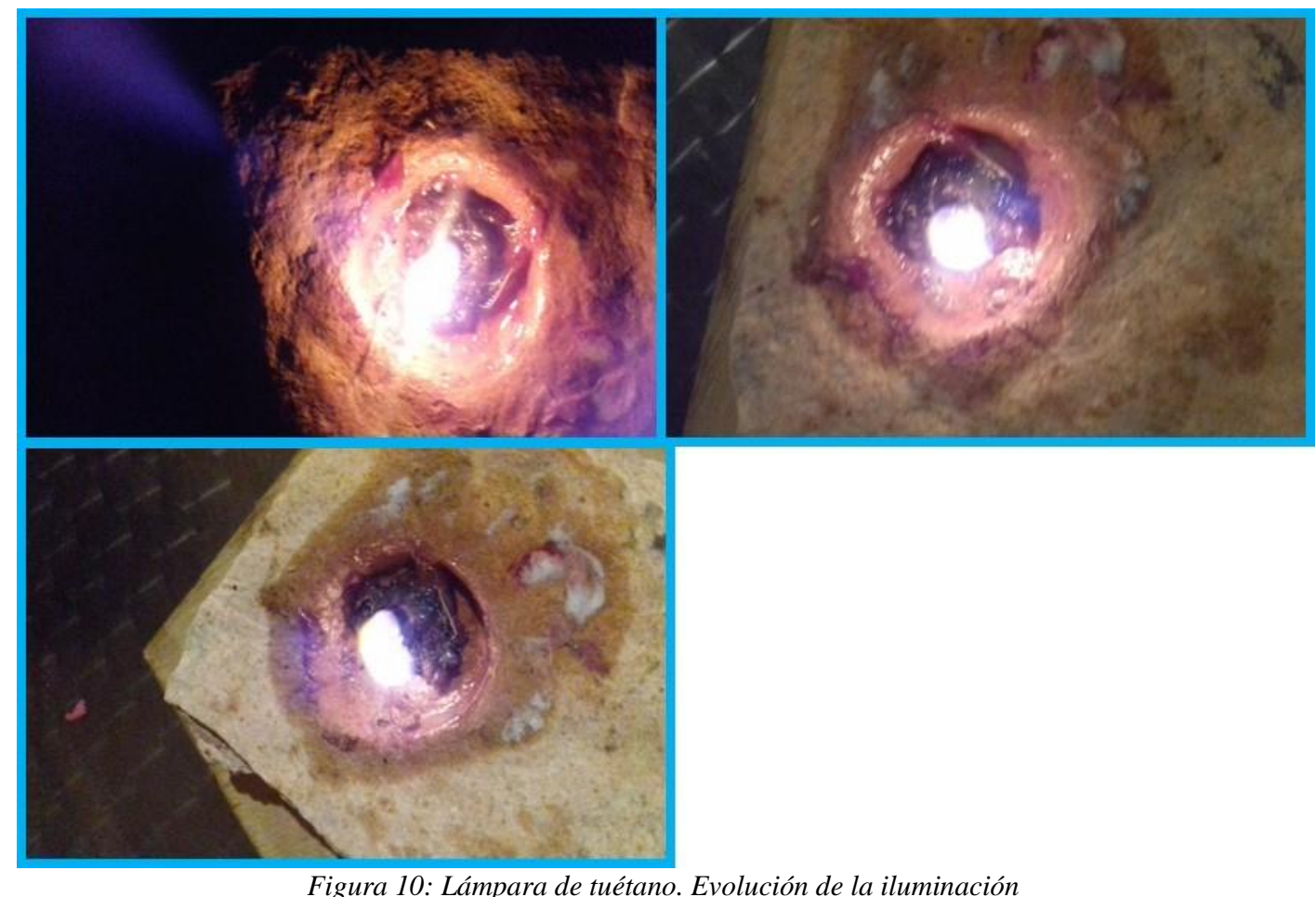

\subsection{Tercer experimento}

La lámpara de sebo al inicio del experimento da una luz más blanca con 0’15 1x de intensidad lumínica. A la hora hay una llama muy pequeña que mantiene otra media hora hasta que se apaga (Fig. 11).

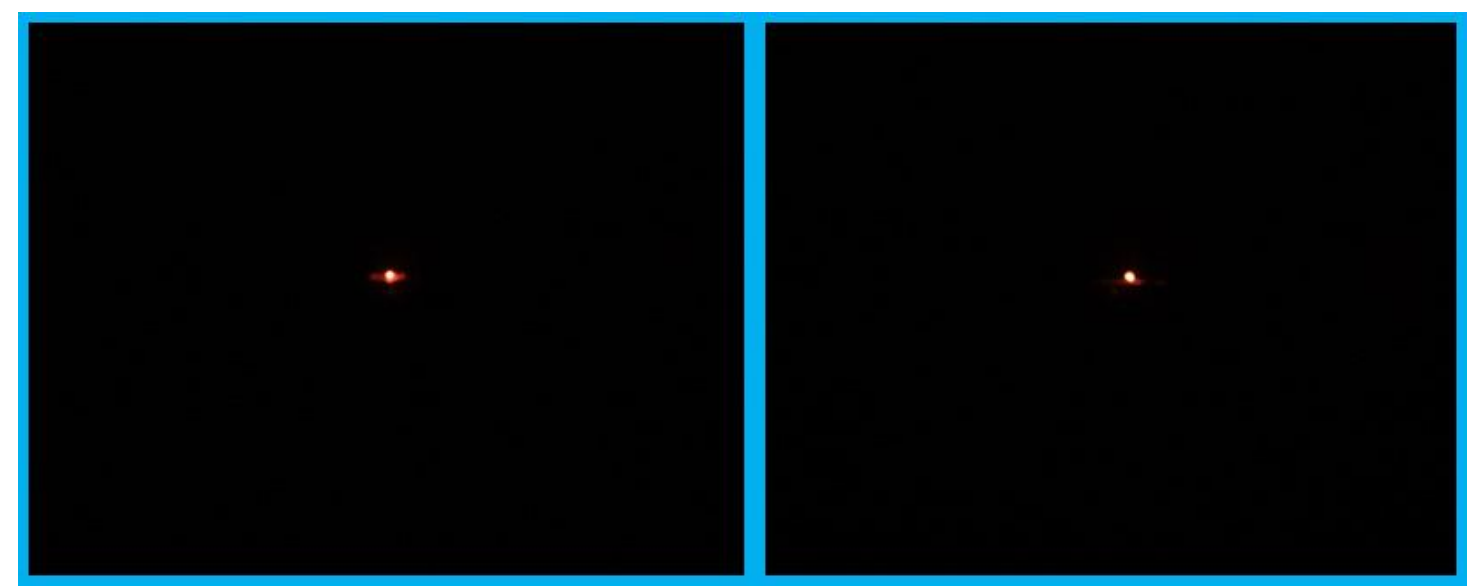

Figura 11. Lámpara de sebo. Evolución de la iluminación

\section{CONCLUSIONES}

Se deduce de esta investigación con respecto a los resultados obtenidos de luminosidad y duración, que el combustible idóneo es el tuétano, seguido de la cera de abeja y del sebo. Es necesario tener en cuenta el tipo de material empleado en la fabricación de la mecha, así como la manera de fabricación. El tuétano, al ser un elemento más graso, genera un aceite que facilita su proceso, llegando a consumir totalmente la cantidad empleada en su proceso. 
Ahora bien, además de lo expuesto anteriormente con respecto a la mecha, al no haber realizado el experimento con los otros dos combustibles (la cera y el sebo) en estado líquido, no se puede concluir si los resultados habrían sido diferentes en ese caso.

La eficacia del tuétano como combustible se ha visto favorecida, en un soporte cerrado, por la mecha y su colocación vertical, produciendo unos resultados equilibrados en luminosidad y duración de la llama, que produce una lámpara eficaz.

\section{Bibliografía}

DE BEAUNE, S. A. (2000): "Les techniques d'éclairage paléolitiques: un bilan". Paleo, 12: 19-27.

DE BEAUNE, S. A. (1987): Lampes et godets au paléolithique, Éditions du CNRS, Paris.

CASO, G. (2011): “Cueva de Ardales (Málaga): el 'Homo sapiens' utilizó cera de abeja para hacer lámparas, según su estudio". En Terrae Antiguae [Blog]: https://terraeantiqvae.com/group/prehistoria/forum/topics/cueva-de-ardales-malaga-elhomo-sapiens-utilizo-cera-de-abeja-par [Último acceso: 29/05/2019].

CANTALEJO, P., ESPEJO, M. M., RAMOS, J. y WENIGER, G. C. (2014): "Elementos de iluminación”. En RAMOS, J., WENIGER, G. C.,CANTALEJO, P. y ESPEJO, M. M. (coords.): Cueva de Ardales (Málaga). Intervenciones arqueológicas 2011-2014. Pp. 119146. Ed. Pinsapar: Benajoán.

CORTÉS, M., SIMÓN, M. D., MORALES-MUÑIZ, A., LOZANO, Mª C., VERA, J. L. y ODRIOZOLA, C. (2016): "La caverna iluminada: una singular lámpara gravetense arroja luz sobre el arte parietal de la cueva de La Pileta (Benaoján, Málaga)". Trabajos de Prehistoria, 73 (1): 115-127.

DIARIO DE LOS YACIMIENTOS DE LA SIERRA DE ATAPUERCA [Online] "Una lámpara de tuétano". En DÍEZ, C. (coord.): Origen. Cuadernos de Atapuerca. Diario de los yacimientos de la sierra de Atapuerca. Arqueología experimental, 13: 11. www.diariodeatapuerca.net/Lamparadetuetano.pdf [Último acceso 29/05/2019].

DE LA RASIlla, M., DUARTE, E., SANTAMARÍA, D., MARTÍNEZ, L., FERNÁNDEZ, J., RODRÍGUEZ, V. y FORTEA, F. (2010): “Licnología paleolítica: las lámparas de las cuevas de Llonín y el Covarón (Asturias)”. Zephyrus, LXV: 103-116

MEDINA, M.Á., CRISTO, A., ROMERO, A. y SANCHIDRIÁN, J. L. (2012): “Otro punto de luz. Iluminación estática en los 'santuarios' paleolíticos: el ejemplo de la Cueva de Nerja (Málaga, España)". En CLOTTES, J. (dir.) : L'art pléistocène dans le monde I Pleistocene art of the world / Arte pleistoceno en el mundo, Actes du Congrès IFRAO, Tarascon-sur-Ariège, septembre 2010, Symposium Art pléistocène en Europe. $\mathrm{N}^{\circ}$ spécial de Préhistoire, Art et Sociétés, Bulletin de la Société Préhistorique Ariège-Pyrénées, LXV-LXVI, 2010-2011, CD: 105-121.

PÉREZ, E., y MUÑOZ DOMÍNGUEZ, D. (2015): Los combustibles en las lámparas del Paleolítico Superior. Trabajo de curso inédito. Universidad Autónoma de Madrid. Departamento de Prehistoria y Arqueología 
